\title{
Protective Effects of Sesamol on the Brains of Rats with Focal Cerebral Ischemia-Reperfusion Injury
}

\author{
Guannan X, Xiujuan G, Xin L, Yujue Z, Zhaoyong \\ $L$, Ximeng $C^{*}$ and Fengzhen $L^{*}$ \\ Liaocheng People's Hospital, Medical College of \\ Liaocheng University, Liaocheng Dongchangfu Hospital, \\ Liaocheng, P. R. China \\ *Corresponding author: Chen Ximeng, Liaocheng \\ People's Hospital, Medical College of Liaocheng \\ University, Liaocheng Dongchangfu Hospital, Liaocheng, \\ 252000, P. R. China
}

Liu Fengzhen, Liaocheng People's Hospital, Medical College of Liaocheng University, Liaocheng Dongchangfu Hospital, Liaocheng, 252000, P. R. China

Received: April 16, 2021; Accepted: May 01, 2021; Published: May 08, 2021

\begin{abstract}
Stroke is an acute cerebrovascular event associated with brain tissue injury, representing the most common cause of death. Thrombolysis and recanalization are the principal treatment modalities for ischemic stroke. Some patients experience cerebral ischemia-reperfusion injury following treatment. A previous study established that sesamol is effective in reducing risk factors for stroke. Here, we aimed to investigate the protective effect of sesame phenol on cerebral ischemia-reperfusion injury. A total of $72 \mathrm{SD}$ rats were randomly divided into a sham group, cerebral ischemia-reperfusion (MCAO) group, cerebral ischemia-reperfusion with low dose sesame phenol (MCAO+sesamol A) group and cerebral ischemia-reperfusion with high dose sesame phenol (MCAO + sesamol B) group. After cerebral ischemia had been induced for $2 \mathrm{~h}$ and reperfusion conducted for $24 \mathrm{~h}$, the volume of cerebral infarction, the degree of cerebral edema and the neurological deficit scores were tested. The results showed sesamin improved the neurological deficit score in a dose-dependent manner, reduced the volume of cerebral infarction, degree of cerebral edema. Prophylactic treatment with sesame phenol provided neuroprotective effects on cerebral ischemia-reperfusion injury.
\end{abstract}

Keywords: Cerebral ischemia-reperfusion injury; Sesamol; Neuroprotective effect; Cell apoptosis

\section{Introduction}

Stroke is an acute cerebrovascular event associated with brain tissue injury. Stroke represents the most common cause of severe permanent disability and the second most common cause of death and dementia. Thrombolysis and recanalization are the principal treatments for ischemic stroke. However, the time window for treatment of recombinant Plasminogen Activator (rtPA) thrombolysis is narrow [1], and requirements for its application strict. Due to specific indications and contraindications, only $2 \%$ to $5 \%$ of stroke patients have the opportunity to receive this form of treatment, even if the probability of success in patients with Cerebral Ischemia Reperfusion Injury (CIRI) is up to 50\% [2]. The pathophysiological mechanisms that result in CIRI are complex, with multiple lesion cascades involved that form a complex network of pathological mechanisms. A number of anti-inflammatory agents and antioxidants have demonstrated particular therapeutic effects in animal experiments, but the results have not been replicated in multiple clinical trials. An important reason is that the mechanisms of action of these drugs encompasses only a fraction of the many mechanisms involved in stroke, rather than representing a multitarget, comprehensive treatment for disease networks. In addition, a number of drugs cannot provide the expected clinical effect due to particular toxic side effects. Therefore, in recent years, the search for a natural plant with active ingredients having multiple biological activities has been an intense focus of research interest, in order to achieve a multi-target and multi-channel treatment for stroke without toxic side effects, so as to provide a broader range of possibilities for the development of novel neuroprotective agents.
Sesamol (also known as 5-hydroxy-1, 3-benzodioxole), a natural extract of flax, is an aromatic component of sesame oil and a principal degreased antioxidant. After passing through the blood-brain barrier, it exhibits a variety of biological properties, being anti-inflammatory, an anti-oxidant, antihypertensive, and reducing apoptosis and lowering lipids [3]. It has recently been demonstrated that sesame phenol has neuroprotective properties, having the capability to maintain the integrity of cells in the neurovascular unit, protect the blood-brain barrier [4], and enhance cognitive function [5]. However, the effects of sesame phenol on acute cerebral ischemia-reperfusion injury have rarely been reported. If would be of considerable clinical significance if sesame phenol were demonstrated to have neuroprotective properties with multiple targets and dimensions. In this study, SD rats in which occlusion of the middle cerebral artery were used to investigate the effects of sesame phenol on CIRI.

\section{Materials and Methods}

\section{Materials}

Experimental animals and groups: Male SD rats (body weight: 170-200 g) that were specific-pathogen free (SPF) were purchased from Shandong Jinfeng Experimental Animal Co., Ltd. (License No.: SCXK (Lu) 2014 0006). The procedures involving animals and their care were approved by the Animal Care and Use Committee of Liaocheng People's Hospital (Grant No.: LY20160916). The SD rats were randomly allocated into one of 4 equal groups according to a random number table method: sham surgery (sham) group, focal middle cerebral artery occlusion (MCAO) group; MCAO+sesamol group $\mathrm{A}$ and $\mathrm{MCAO}+$ sesamol group $\mathrm{B}$, the latter two receiving $\mathrm{MCAO}$ surgery, but with sesame phenol (98\% purity) at doses of $5 \mathrm{mg} \cdot \mathrm{kg}$ 
${ }^{1} \cdot \mathrm{d}^{-1}$ and $25 \mathrm{mg} \cdot \mathrm{kg}^{-1} \cdot \mathrm{d}^{-1}$, injected intraperitoneally for 7 days prior to MCAO. The sham and MCAO groups were injected intraperitoneally continuously in the same manner but with physiological saline as control treatments.

Principal reagents and equipment: Sesamin (BSZH-Z-021, Sigma-Aldrich Co. Ltd), TTC solution (T8877-5G, SigmaAldrich Co. Ltd), DAB chromogenic kit (Nanjing Institute of Bioengineering), TUNEL staining kits, Caspase- 3 antibody kits and SABC immunohistochemical staining kits were purchased from Wuhan Bude Biotechnology Engineering Co., Ltd. The following reagents and equipment were sourced as follows: goat anti-rabbit IgG/horseradish peroxidase (ZB2303, Beijing Zhongshan Jinqiao Biotechnology Co., Ltd.), laser doppler blood flow monitor (PeriFlux, Stockholm, Sweden), paraffin slicer (Shandon 325, Shandon, UK), embedding box (Jiangsu Shitai Experimental Equipment Co., Ltd.), digital camera (Olympus, Japan) and image analysis system (ImagePro Plus 6.0).

\section{Methods}

Establishing the MCAO model: The MCAO model consisted of the wire plug method described by Chen [6]. The wire plug consisted of a $0.15 \mathrm{~mm}$ diameter, $4 \mathrm{~cm}$ long sterile nylon filament thread $(20 \mathrm{~mm}$ of one end coated with polylysine) and the other end coated with silica gel. Rats were anesthetized with $3 \%$ isoflurane and maintained with $2 \%$ isoflurane in a gas mixture of $5 \% \mathrm{CO}_{2}$ and $95 \% \mathrm{O}_{2}$. During the entire procedure, temperature was maintained at $37^{\circ} \mathrm{C}$ using a thermostatically controlled heated blanket. For the MCAO model, blood flow in the obstructed artery was monitored using a doppler blood flow monitor in real time. When blood flow had decreased by more than $85 \%$, the experiment was deemed to have begun. At this stage, the color of the iris became lighter, then white, and the skin was sutured layer by layer, with no active bleeding observed. The nylon filament was tightened for $2 \mathrm{~h}$, and then reperfusion allowed for $24 \mathrm{~h}$ The middle cerebral artery in the rats in the sham group was exposed but not subjected to ischemia-reperfusion surgery. During surgery, if a rat developed dyspnea or excessive bleeding, there were replaced by an additional rat, such that the number of animals in each group was 18. The vital signs of the rats were monitored during the perioperative period, and HR, RR, $\mathrm{SPO}_{2}$ and $\mathrm{T}$ recorded $5 \mathrm{~min}$ prior to MCAO, $5 \mathrm{~min}$ afterward, $5 \mathrm{~min}$ before reperfusion, and $5 \mathrm{~min}$ after reperfusion.

Evidence that MCAO was successfully performed included development of Horner syndrome on the same side as ischemia, with hemiplegia of the contralateral forelimb following recovery from anesthesia.

Neurological deficit score: After $2 \mathrm{~h}$ of cerebral ischemia and $24 \mathrm{~h}$ of reperfusion, the neurological deficit of the rats using criteria from the Christa report [7] were recorded, in a blinded-fashion. The score comprises 6 tests: 1) Autonomic movement of the rats; 2) Symmetry of limb movement; 3) Forelimb extension function; 4) Climbing movement; 5) Proprioceptive function; Each test was scored from 0 to 3 , with a minimum neurological score of 0 and a maximum of 18 points. Lower scores represented more severe neurological dysfunction.

Measurement of volume of cerebral infarction: Six rats in each group were randomly injected with $3 \%$ isoflurane and maintained with $2 \%$ isoflurane in a gas mixture of $5 \% \mathrm{CO}_{2}$ and $95 \% \mathrm{O}_{2}$ for anesthesia. The rat was quickly decapitated then the head placed on ice, after which blood was washed away and the head frozen at $-20^{\circ} \mathrm{C}$ in a freezer for $30 \mathrm{~min}$. The volume percentage of cerebral infarction was calculated according to the method described by Qiu $\mathrm{ZD}$ : the percentage of cerebral infarction volume $(\%)=$ [total cerebral infarction volume - (volume of right brain hemisphere - volume of left hemisphere)] / total volume of left brain tissue $\times 100 \%$ [8]

Calculation of water content in ischemic brain tissue: After cerebral ischemia for $2 \mathrm{~h}$ and reperfusion for $24 \mathrm{~h}, 6$ rats in each group were randomly selected for decapitation, and the water content of the brain tissue measured using the dry and wet weighing method. The weight was immediately measured, representing the wet weight (G1); brain tissue was wrapped in aluminum foil, baked within a constant temperature drying oven at $110^{\circ} \mathrm{C}$ until the weight had equilibrated, representing the dry weight $(\mathrm{G} 2)$. Brain tissue water content $=[(\mathrm{G} 1$ G2) / G1] $\times 100 \%$.

Statistical methods: SPSS v16.0 software was used to conduct statistical analysis on the data. A Kolmogorov-Smirnov analysis was used for the testing of normality, the Levene method for assessing homogeneity of variance, and a comparison of multiple sets of data performed by one-way ANOVA. Multiple comparisons of sample means were evaluated using a Tukey test, and a nonparametric rank sum test used when there was no difference in variance. The vital signs of the rats were analyzed by repeated measures of variance, and the Bonferroni method used for comparisons between groups. The test level was $\alpha=0.05$, with $\mathrm{P}<0.05$ considered statistically significant. Graph pad Prime 5.0 software was used to plot graphs.

\section{Results}

\section{Comparison of general information}

Modeling: In the present study, 82 SD rats were used, of which 8 rats were excluded from the cerebral ischemia-reperfusion injury model. Of these, 5 died due to subarachnoid hemorrhage, 2 suffered severe cerebral edema and respiratory depression occurred in 1 when the vagus nerve was isolated. No deaths occurred in the SD rats in the sham group. The success rate of the modeling was $84 \%$, with mean duration of $22 \mathrm{~min}$.

Regional cerebral blood flow in each group: Before MCAO, there was no significant difference in the regional Cerebral Blood Flow

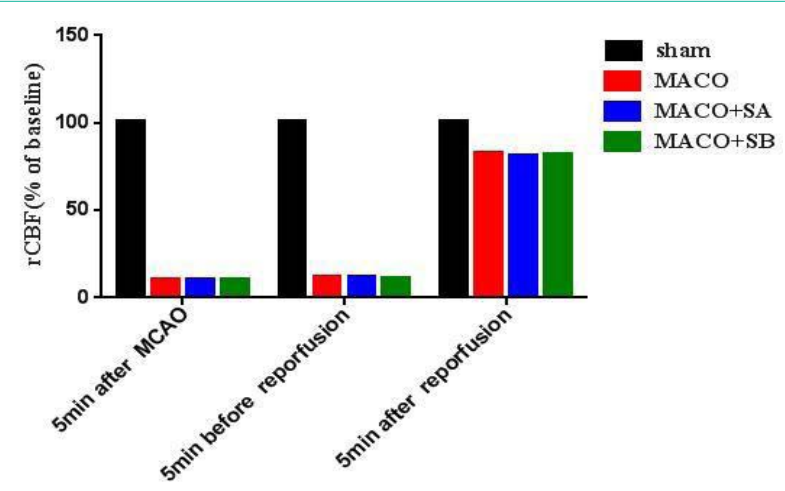

Figure 1: Changes of cerebral blood flow before and after occlusion of middle cerebral artery and reperfusion in rats. 
Table 1: Comparison of general data of four groups of rats.

\begin{tabular}{|c|c|c|c|c|}
\hline & Sham $(n=18)$ & MCAO $(n=18)$ & MCAO+sesamol A $(n=18)$ & MCAO+sesamol B $(n=18)$ \\
\hline \multicolumn{5}{|c|}{ Before MCAO (5min) } \\
\hline HR (time/min) & $365 \pm 14$ & $376.4 \pm 7.6$ & $379.8 \pm 10.5$ & $374.2 \pm 11$ \\
\hline RR (time/min) & $62.5 \pm 4.3$ & $60.6 \pm 5.1$ & $64.1 \pm 1.4$ & $63.9 \pm 4.0$ \\
\hline $\mathrm{SPO}_{2}(\%)$ & $99.3 \pm 0.2$ & $99.1 \pm 0.2$ & $98.1 \pm 0.3$ & $99.7 \pm 0.1$ \\
\hline $\mathrm{T}\left({ }^{\circ} \mathrm{C}\right)$ & $36.7 \pm 0.1$ & $36.9 \pm 0.3$ & $37.3 \pm 0.1$ & $36.5 \pm 0.2$ \\
\hline \multicolumn{5}{|c|}{ After MCAO (5min) } \\
\hline HR (time/min) & $369 \pm 10$ & $385.3 \pm 5.6$ & $378.7 \pm 7.8$ & $375 \pm 8.4$ \\
\hline RR (time/min) & $62.7 \pm 5.3$ & $63 \pm 5.1$ & $65.4 \pm 1.6$ & $68.4 \pm 1.5$ \\
\hline $\mathrm{SPO}_{2}(\%)$ & $99.2 \pm 0.3$ & $99.1 \pm 0.3$ & $98.9 \pm 0.2$ & $99.4 \pm 0.2$ \\
\hline $\mathrm{T}\left({ }^{\circ} \mathrm{C}\right)$ & $36.4 \pm 0.2$ & $36.3 \pm 0.3$ & $36.5 \pm 0.2$ & $37.3 \pm 0.1$ \\
\hline \multicolumn{5}{|c|}{ Before reperfusion (5min) } \\
\hline HR (time/min) & $379 \pm 10$ & $390 \pm 5.8$ & $403 \pm 8.5$ & $400.2 \pm 6.6$ \\
\hline RR (time/min) & $72.5 \pm 2.5$ & $70.5 \pm 1.0$ & $72.8 \pm 1.5$ & $71.3 \pm 1.6$ \\
\hline $\mathrm{SPO}_{2}(\%)$ & $99.3 \pm 0.1$ & $99.4 \pm 0.1$ & $99.2 \pm 0.1$ & $99.0 \pm 0.2$ \\
\hline $\mathrm{T}\left({ }^{\circ} \mathrm{C}\right)$ & $36.2 \pm 0.2$ & $37.8 \pm 0.3$ & $38.0 \pm 0.1$ & $38.1 \pm 0.2$ \\
\hline \multicolumn{5}{|c|}{ After reperfusion (5min) } \\
\hline HR (time/min) & $387 \pm 9$ & $401.4 \pm 8.4$ & $397.1 \pm 5.5$ & $394 \pm 10$ \\
\hline RR (time/min) & $72.2 \pm 3.2$ & $72.6 \pm 1.4$ & $72.1 \pm 3.1$ & $70.5 \pm 1.5$ \\
\hline $\mathrm{SPO}_{2}(\%)$ & $99.3 \pm 0.1$ & $98.7 \pm 0.4$ & $99.5 \pm 0.1$ & $99.7 \pm 0.1$ \\
\hline $\mathrm{T}\left({ }^{\circ} \mathrm{C}\right)$ & $73.2 \pm 3.2$ & $37.3 \pm 0.1$ & $37.5 \pm 0.2$ & $37.6 \pm 0.1$ \\
\hline
\end{tabular}

(rCBF) compared with $5 \mathrm{~min}$ after MCAO, $5 \mathrm{~min}$ before reperfusion, and $5 \mathrm{~min}$ after reperfusion (Figure 1 ).

General condition of the rats: After ischemia for $2 \mathrm{~h}$ and reperfusion for $24 \mathrm{~h}$, it was observed that the rats in the MCAO group exhibited significantly decreased levels of feeding and drinking of water, their fur was dull, the right limb was weak, the circle was turned to the right, and they exhibited only limited consciousness. Rats in the sham group did not significantly change the quantities that they drank or ate compared with prior to surgery, and their fur was shiny and mental state good. Feeding and drinking of the rats in the $\mathrm{MCAO}+$ sesamol A and MCAO+sesamol B groups were less affected than those in the MCAO group. There were no significant differences in $\mathrm{HR}, \mathrm{RR}, \mathrm{SPO}_{2}$, and $\mathrm{T}$ recorded $5 \mathrm{~min}$ before MCAO, $5 \mathrm{~min}$ after $\mathrm{MCAO}, 5 \mathrm{~min}$ before reperfusion and $5 \mathrm{~min}$ after reperfusion (Table $1)$.

Neurological deficit scores: After 24 hours of reperfusion, neurological deficits were scored according to Garcia scores. The results demonstrated that there was clear neurological dysfunction due to middle cerebral artery occlusion and reperfusion. The MCAO group had the most serious neurological deficit (13.13 \pm 0.83 ) compared with neurological function scores of the sham group $(18 \pm 0)$ which were normal. The scores of the MCAO+sesamol A and $\mathrm{MCAO}+$ sesamol B groups were significantly higher $(15.57 \pm 0.45$ vs. $13.13 \pm 0.83, \mathrm{P}<0.05 ; 16.88 \pm 0.54$ vs. $13.13 \pm 0.83, \mathrm{P}<0.05)$ than the $\mathrm{MCAO}$ group, and the $\mathrm{MCAO}+$ sesamol $\mathrm{B}$ group had significantly higher scores than the MCAO+sesamol A group (Figure 2).

Comparison of volume of cerebral infarction: Following cerebral ischemia for $2 \mathrm{~h}$ and reperfusion for $24 \mathrm{~h}$, the three groups

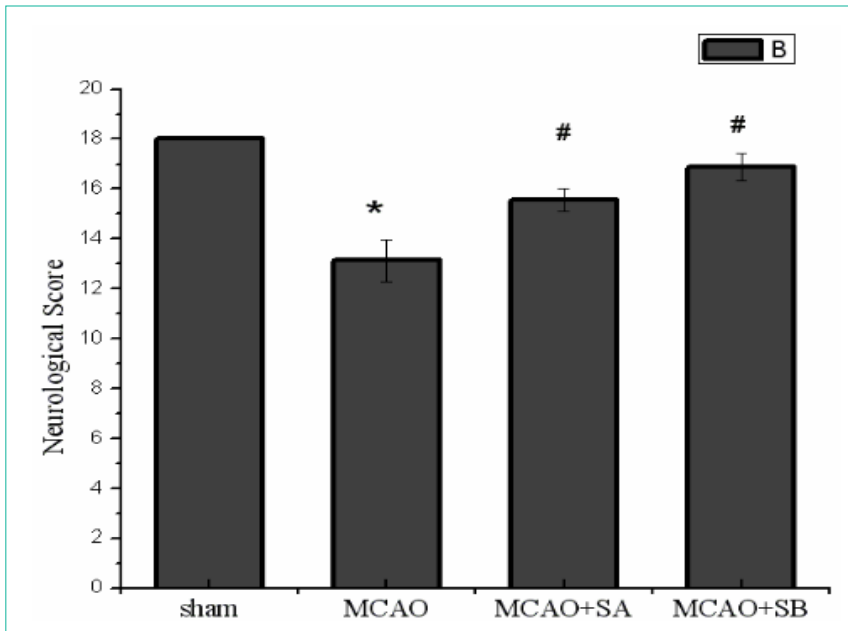

Figure 2: Comparison of neurological deficit scores. Remarks: *Indicates comparison with the sham group, $\mathrm{P}<0.05$; "Indicates comparison with the MCAO model group, $\mathrm{P}<0.05$.

in which MCAO was performed experienced significant infarction $(\mathrm{P}<0.05)$, compared with the sham group. The volume of cerebral infarction in the $\mathrm{MCAO}+$ sesamol $\mathrm{A}$ and $\mathrm{MCAO}+$ sesamol $\mathrm{B}$ groups was significantly lower than that of the MCAO group $(29.37 \% \pm 1.8 \%$ vs. $40.31 \% \pm 2.3 \%, 19.58 \% \pm 1.22 \%$ vs. $40.31 \% \pm 2.3 \%, \mathrm{P}<0.01)$. The largest difference was in the MCAO+sesamol B group (Figure 3).

The results of brain tissue sections of group A after TTC staining showed that red represents healthy brain tissue and white represents damaged brain tissue. Comparison of the percentage of cerebral 
A

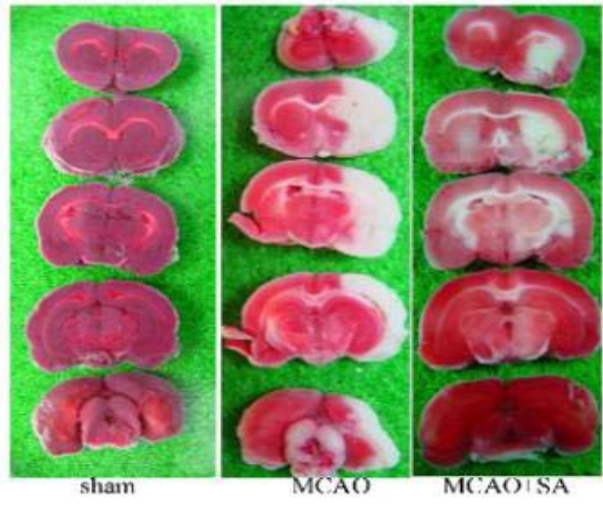

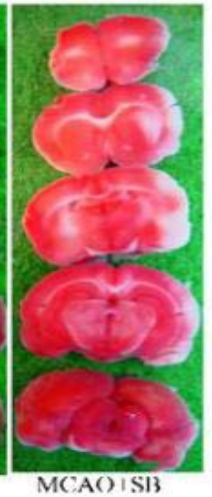

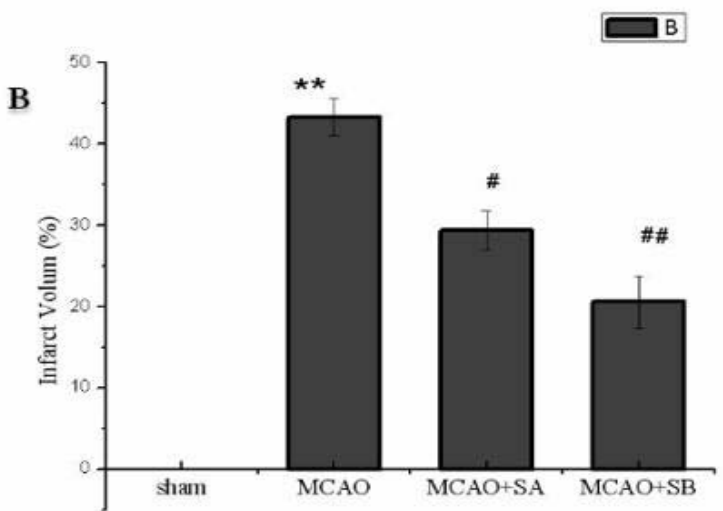

Figure 3: Comparison of cerebral infarction volume in four groups of rats. The effect of MCAO and sesame phenol on the volume of cerebral infarction after ischemia for $2 \mathrm{~h}$ and reperfusion for $24 \mathrm{~h}$.

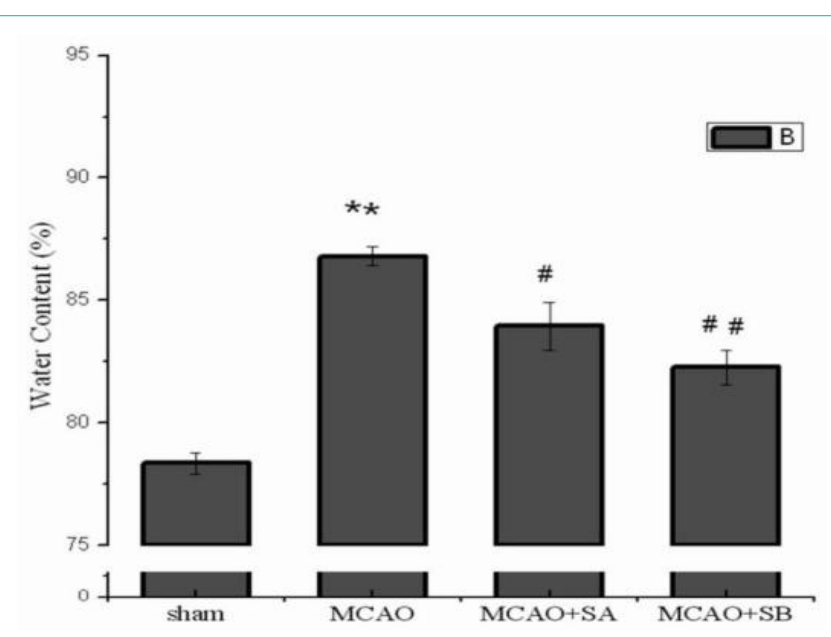

Figure 4: Comparison of brain water content in four groups of rats. Note: "Indicates $\mathrm{P}<0.01$ compared with sham operation group, "Represents $\mathrm{P}<0.05$ compared with MCAO model group, and ${ }^{\#}$ Represents $P<0.01$ compared with MCAO model group.

infarction volume in each group of $\mathrm{B}$. "*indicates $\mathrm{P}<0.01$ compared with sham operation group, "represents $\mathrm{P}<0.05$ compared with MCAO model group, and ${ }^{* *}$ represents $\mathrm{P}<0.01$ compared with MCAO model group.

Change in brain tissue water content: After ischemia for $2 \mathrm{~h}$ and reperfusion for $24 \mathrm{~h}$, no brain edema was observed in the brain tissue of the sham group. Significant cerebral edema was observed in the MCAO group $(86.80 \pm 0.38 \%$ vs. $78.95 \pm 0.44 \%, \mathrm{P}<0.01)$ compared with the sham group. The MCAO+sesamol A and MCAO+sesamol B groups experienced significantly lower levels of brain water content, compared with the MCAO group $(83.95 \pm 0.97 \%$ vs. $86.80 \pm 0.38 \%$, $\mathrm{P}<0.05 ; 82.57 \pm 0.72 \%$ vs. $86.80 \pm 0.38 \%, \mathrm{P}<0.01$, respectively), brain edema more effectively prevented in the $\mathrm{MCAO}+$ sesamol $\mathrm{B}$ group (Figure 4).

\section{Discussion}

In this study, the results indicated that pretreatment with sesame phenol can reduce neurological dysfunction caused by ischemiareperfusion injury, reduce the volume of cerebral infarction and degree of cerebral edema. Pretreatment with sesame phenol, therefore, provided a degree of protection against cerebral ischemia-reperfusion injury, an effect that was dose-dependent, further confirming the neuroprotective effect of sesame phenol.

The present study used the internationally recognized and most commonly used Longa modified suture method to successfully establish the cerebral ischemia-reperfusion injury model. The MCAO model can induce a series of pathophysiological changes similar to human stroke [9]. Compared with SD rats, the volume of cerebral infarction of Wistar rats has been shown to be small, with large infarcts combined with poor stability [10]. Therefore, SD rats were selected for the MCAO animal model. According to our previous experience, if the initial part of the wire plug is covered with polylysine, and the other end coated with silica gel, the cross-section of the cord is round with a smooth surface, ensuring better compatibility and reducing damage to the blood vessel wall. Secondly, the procedure was conducted under a microscope, allowing the surgeon to clearly observe the process of threading the plug into the ICA, reducing the force required when pulling and damage to the blood vessels and nerves, and reducing the risk of subarachnoid hemorrhage caused by arterial injury or Willis rupture. In addition, real-time monitoring of blood flow in the middle cerebral artery in the rat skull surface by laser color doppler ultrasound allowed visualization of the process of blood flow blockage and reperfusion, further improving the success of modeling. Thus, this procedure was more stable and repeatable.

The Garcia neurological deficit score can be used to simply and quickly evaluate neurological dysfunction, both comprehensively and effectively. TTC staining is also an important indicator for judging the success of modeling. The combination of the two is often used as an evaluation of the efficacy of a drug. The results of this study confirmed that sesame phenol improved neurological deficit and reduced cerebral infarction volume caused by cerebral ischemia-reperfusion injury, demonstrating neuroprotective effects consistent with the findings of Kumar [11] and Sonia Angeline M [12]. Neurotoxicity induced by trinitropropane in the rat model of Kumar was pretreated with sesame phenol, which significantly improved the coordination of body weight, spontaneous activity and movement, and a reduction in brain damage. Sonia Angeline M administered sesame phenol $\left(15 \mathrm{mg} \cdot \mathrm{kg}^{-1}\right)$ or naringenin $\left(10 \mathrm{mg} \cdot \mathrm{kg}^{-1}\right)$ orally in a rotenone-induced 
SD rat model for 10 consecutive days. As a result, motor function and body weight increased significantly in the rats. Protein and ubiquitin levels were reduced, indicating successful neuroprotection from the sesaminol.

In 2015, Bo Young Hong [13] found that sesame phenol did not improve forelimb movement and the volume of cerebral infarction in MCAO rats, possibly related to insufficient dosing in that study, using a different timing compared with the present study. In that study, sesame phenol $\left(8 \mathrm{mg} \cdot \mathrm{kg}^{-1} \cdot \mathrm{d}^{-1}\right)$ was administered after establishing the CIRI model for 5 consecutive days. Studies have confirmed that the bioavailability of oral sesame phenol is only $35.5 \% \pm 8.5 \%$ [14]. In addition, the study dose was lower than the minimum dose of the present study, and so the quantity of sesame phenol reaching the brain tissue may have been insufficient, resulting in only a limited improvement in forelimb movement and volume of cerebral infarction, differences that were not significant. However, other studies have observed that sesame phenol significantly improves lower extremity dyskinesia. These data also suggest that sesame phenol can protect against CIRI. In study of the Bo Young Hong, the success rate of the MCAO model was only $50 \%$, and the mortality rate was high. There were only 7 rats in the final treatment group and 8 model groups had smaller sample sizes, possible reasons for the results not being significant.

The destruction of the blood-brain barrier and development of brain edema following CIRI is an important mechanism of brain injury. The severity of cerebral edema is closely related to the prognosis and outcome in patients. In the present study, pretreatment with sesame phenol reduced the degree of edema in brain tissue, although its specific mechanism remains to be further studied. However, previous studies have confirmed that sesame phenol can reduce the expression of ICAM-1 [15], which has a regulatory effect on the MMP family, beneficial for maintenance of the integrity of the extracellular matrix [16], promotion of the expression of microvascular tight junction proteins, and reduction and destruction of the function and structure of the blood-brain barrier [4]. This may be the mechanisms by which sesame phenol reduces brain edema.

There are many limitations to this study. Firstly, the timing of sesame phenol administration in this study prior to injury, and the effects of the timing of other factors on brain protection were not studied. The time window for use of sesame phenol will be investigated in future studies. Secondly, only 2 doses of sesame phenol $(15 \mathrm{mg}$ $\mathrm{mg} \cdot \mathrm{kg}^{-1} \cdot \mathrm{d}^{-1}$ and $\left.25 \mathrm{mg} \cdot \mathrm{kg}^{-1} \cdot \mathrm{d}^{-1}\right)$ were evaluated and their concentration in the blood of the rats was not measured in this study. The optimal dose has therefore yet to be confirmed. In addition, the duration of this study was not sufficiently long to evaluate the sequelae of ischemic stroke during the chronic phase, which will be investigated in future experiments. Therefore, a larger cohort and a greater range of drug doses with different durations will provide more conclusive evidence for the efficacy of sesame phenol.

\section{Conclusion}

In this study, the results indicated that pretreatment with sesame phenol can reduce neurological dysfunction caused by ischemiareperfusion injury, reduce the volume of cerebral infarction and degree of cerebral edema. Pretreatment with sesame phenol, therefore, provided a degree of protection against cerebral ischemia-reperfusion injury, an effect that was dose-dependent, further confirming the neuroprotective effect of sesame phenol.

\section{Acknowledgment}

This work was in part supported by Science Foundation of Shandong Province of China (Grant no: ZR2019PH090) and Medicine and Health Science Technology Development Plan of Shandong Province of China (Grant no: 2017WS503, 2018WSA15023).

\section{References}

1. Furlan AJ. Endovascular therapy for stroke-it is about time. N Engl J Med. 2015; 372: 2347-2349.

2. Shirley R, Ord EN, Work LM. Oxidative Stress and the use of antioxidants in stroke. Antioxidants (Basel). 2014; 3: 472-501.

3. Selvarajan K, Narasimhulu CA, Bapputty R, et al. Anti-inflammatory and antioxidant activities of the nonlipid (aqueous) components of sesame oil: potential use in atherosclerosis. J Med Food. 2015; 18: 393-402.

4. VanGilder RL, Kelly KA, Chua MD, et al. Administration of sesamol improved blood-brain barrier function in streptozotocin-induced diabetic rats. Exp Brain Res. 2009; 197: 23-34.

5. Misra S, Tiwari $\mathrm{V}$, Kuhad $\mathrm{A}$, et al. Modulation of nitrergic pathway by sesamol prevents cognitive deficits and associated biochemical alterations in intracerebroventricular streptozotocin administered rats. Eur J Pharmacol. 2011; 659: 177-186.

6. Chen JL, Yi L, Wang L, et al. Therapeutic benefit of intravenous administration of bone marrow stromal cells after cerebral ischemia in rats. Stroke. 2001; 32: 1005-1011.

7. Christa Thoene-Reineke, Kay Rumschüssel, Kristin Schmerbach, et al. Prevention and intervention studies with telmisartan, ramipril and their combination in different rat stroke models. Plos One. 2011; 6: e23646.

8. Benedek A, Móricz K, Jurányi Z, et al. Use of TTC staining for the evaluation of tissue injury in the early phases of reperfusion after focal cerebral ischemia in rats. Brain Res. 2006; 1116: 159-165.

9. Sato-Maeda M, Fujimura M, Kanoke A, et al. Transient middle cerebral artery occlusion in mice induces neuronal expression of RNF213, a susceptibility gene for moyamoya disease. Brain Res. 2016; 1630: 50-55.

10. Culman J, Nguyen-Ngoc M, Glatz T, et al. Treatment of rats with pioglitazone in the reperfusion phase of focal cerebral ischemia: a preclinical stroke trial. Exp Neurol. 2012; 38: 243-253.

11. Kumar P, Kalonia $\mathrm{H}$, Kumar A. Sesamol attenuate 3-nitropropionic acidinduced Huntington-like behavioral, biochemical, and cellular alterations in rats. J Asian Nat Prod Res. 2009; 11: 439-450.

12. Sonia AM, Sarkar A, Anand K, et al. Sesamol and naringenin reverse the effect of rotenone-induced PD rat model. Neuroscience. 2013; 254: 379.

13. Hong BY, Kim JS, Lee KB, et al. The effect of sesamol on rats with ischemic stroke. J Phys Ther Sci. 2015; 27: 1771-1773.

14. Kakkar V, Mishra AK, Chuttani K, et al. Delivery of sesamol-loaded solid lipid nanoparticles to the brain for menopause-related emotional and cognitive central nervous system derangements. Rejuvenation Res. 2011; 14: 597604.

15. Wu WH, Wang SH, Kuan II, et al. Sesamin attenuates intercellular cell adhesion molecule-1 expression in vitro in TNF-alpha-treated human aortic endothelial cells and in vivo in apolipoprotein-E-deficient mice. Mol Nutr Food Res. 2010; 54: 1340-1350.

16. Periasamy S, Mo FE, Chen SY, et al. Sesamol attenuates isoproterenolinduced acute myocardial infarction via inhibition of matrix metalloproteinase-2 and -9 expression in rats. Cell Physiol Biochem. 2011; 27: 273-280. 